\title{
ПОВЫШЕНИЕ ЭНЕРГОЭФФЕКТИВНОСТИ ПРОИЗВОДСТВА ЗА СЧЕТ ПРИМЕНЕНИЯ СИЛОВЫХ ФИЛЬТРОВ ВЫСШИХ ГАРМОНИК
}

\author{
Нос Олег Викторович',
}

nos@corp.nstu.ru

Востриков Анатолий Сергеевич',
vostrikov@sintez.nstu.ru

Штанг Александр Александрович',

shtang@corp.nstu.ru

\author{
Малявко Екатерина Юрьевна', \\ arteb@smc.nstu.ru \\ 1 Новосибирский государственный технический университет, \\ Россия, 630073, г. Новосибирск, пр. К. Маркса, 20.
}

Актуальность. В настоящее время все большее количество технологических процессов и производств в нефтегазовой отрасли реализуется на базе высокопроизводительного оборудования с полупроводниковыми преобразователями электрической энергии, в число которых, например, входят частотно-регулируемые электроприводы переменного тока или бесперебойные источники питания, которые относятся к классу нелинейных нагрузок и могут вызывать большое количество негативных явлений в работе распределительных сетей, включающих в себя несимметрию фазных напряжений и токов, резонансные процессы, тепловые потери в нейтральном проводе, перегрев двигателей и трансформаторов и т. Д. Для улучшения электромагнитной совместимости различного рода электроприемников в составе промышленных систем электроснабжения довольно часто используют пассивные конденсаторные батареи для коррекции коэффициента мощности, которые малоэффективны в случае нелинейных процессов. Кроме этого, данный тип компенсационных устройств продолжает потреблять реактивную энергию при отсутствии каких-либо нагрузок и не удовлетворяет современным требованиям в области энергосбережения. Описанные выше недостатки в работе трехфазных систем переменного тока требуют разработки новых схемотехнических решений, методов анализа энергетических процессов и синтеза алгоритмов силовой фильтрации, позволяющих обеспечить нормированные показатели качества электрической энергии в распределительных сетях низкого и среднего классов напряжения вне зависимости от конкретного вида электрических цепей нагрузки.

Цель исследования заключается в комплексном анализе существующих технических решений, направленных на повышение электромагнитной совместимости распределительных сетей, а также в описании основных ограничений в работе силовых фильтрокомпенсирующих устройств применительно к промышленным объектам минерально-сырьевого комплекса; в разработке базовых структур активных силовых фильтров с пониженными требованиями по производительности, объему памяти и быстродействию программно-аппаратной части системы управления, практическое применение которых обеспечивает нормированное качество электрической энергии при изменении режимов работы технологического оборудования или конфигурации питающей линии.

объекты: автономные или децентрализованные электроэнергетические системы переменного тока с преобразовательными устройствами силовой электроники и частотно-регулируемыми электроприводами, а также элементами силовой цепи с нелинейными характеристиками, например, реакторами или трансформаторами с насыщенными сердечниками, в которых присутствуют значительные искажения в мгновенной форме трехфазных сигналов; пассивные и активные силовые фильтры высших гармоник.

Методы: некоммутативная алгебра кватернионов; четырехмерное гиперкомплексное пространство; методы спектрального анализа и разложения в ряд Фурье.

Результат: краткий обзор различных подходов и технических средств к повышению качества электрической энергии в системах электроснабжения промышленных объектов минерально-сырьевого сектора, а также системы управления, в рамках которых достигается синусоидальный закон изменения во времени сетевых токов с нулевым или опережающим/отстающим угловым сдвигом при одновременном соблюдении условия симметрии по мгновенным значениям.

\section{Ключевые слова:}

Нелинейные электромагнитные процессы, энергоэффективность, силовые фильтрокомпенсирующие устройства, последовательно-параллельные активные силовые фильтры, гиперкомплексные числа.

\section{Введение}

В настоящее время одним из наиболее эффективных путей существенного увеличения текущих объемов добычи и переработки полезных ископаемых является глубокая модернизация существующего электрооборудования технологических машин на базе современных полупроводниковых преобразователей электрической энергии с микропроцессорным управлением, установленная мощ- ность которых может достигать десятков МВА [1]. Данный класс устройств силовой электроники, в силу протекающих в них существенно нелинейных электромагнитных процессов, вызывает амплитудно-фазовую асимметрию и искажения в мгновенной форме электрических сигналов распределительной сети [2], которые сопровождаются дополнительными потерями в трансформаторах и коммутационной аппаратуре, неравномерной за- 
грузкой фаз линий электропередачи, интенсивным старением изоляции электрических машин и т. д.

Для исключения влияния указанных выше негативных факторов на энергоэффективность систем электроснабжения объектов горнодобывающей или нефтегазовой инфраструктуры, что особенно актуально в случае их ограниченной пропускной способности или большой протяженности, применяются специальные фильтрокомпенсирующие устройства (ФКУ) пассивного или активного принципа действия [3]. Данная статья посвящена анализу современных технических средств силовой фильтрации высших гармоник для диапазона низкого и среднего класса напряжений, позволяющих значительно снизить величину среднеквадратических потерь в общем составе активной мощности, затрачиваемой на реализацию производственного процесса.

\section{Пассивные фильтрокомпенсирующие устройства}

Пассивные ФКУ, которые в зависимости от конкретного вида частотных характеристик подразделяются на резонансные RLC-контуры, фильтры верхних частот или их различные совместные комбинации, осуществляют шунтирование источника с суммарным операторным сопротивлением $Z_{s}(p)$ по цепи прохождения пульсационных составляющих тока нагрузки $i_{h}$ в соответствии со следующим соотношением [4]:

$$
i_{f}=\frac{Z_{s}(p)}{Z_{s}(p)+Z_{f}(p)} i_{h},
$$

$f$ - нижний индекс, показывающий на принадлежность параметра или переменной к ФКУ.

Как видно из последней формулы, в идеализированном случае должно выполняться предельное требование

$$
\frac{Z_{s}(p)}{Z_{s}(p)+Z_{f}(p)} \rightarrow 1,
$$

которое на практике принимается равным 0,995 [5] и имеет место при $Z_{s}(p)>>Z_{f}(p)$.

На рис. 1 изображены упрощенные принципиальные схемы наиболее распространенных в энергетическом комплексе топологий пассивных ФКУ второго порядка, которые настраиваются на частоту фильтрации

$$
\omega_{0}=\frac{1}{\sqrt{L_{f} C_{f}}},
$$

а их динамические свойства описываются следующими соотношениями [6]:

- пассивный резонансный контур (рис. $1, a$ )

$$
Z_{f}(p)=\frac{C_{f} L_{f} p^{2}+C_{f} R_{f} p+1}{C_{f} p},
$$

- силовой фильтр высокочастотных гармоник (рис. 1, б)

$$
Z_{f}(p)=\frac{C_{f} L_{f} p^{2}+T_{f} p+1}{C_{f} p\left(T_{f} p+1\right)},
$$

где $T_{f}=L_{f} R_{f}^{-1}-$ постоянная времени.
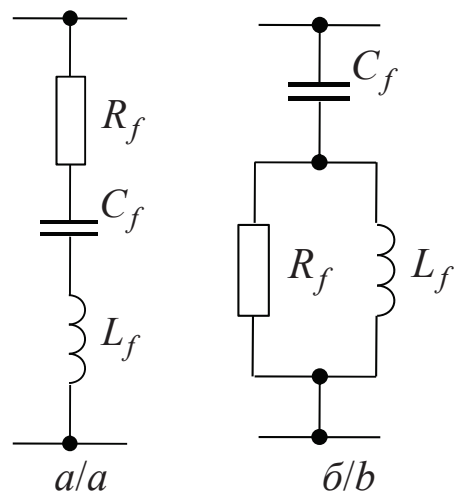

Pис. 1. Пассивные ФКУ второго порядка

Fig. 1. Passive second-order filters

Качественные показатели работоспособности данного типа ФКУ в частотной области однозначно определяются добротностью, которая зависит от выбора $R_{f}$ и для RLC-цепи с (2) вычисляется по формуле [7]:

$$
Q=\omega_{0} \frac{L_{f}}{R_{f}}=\frac{1}{R_{f}} \sqrt{\frac{L_{f}}{C_{f}}},
$$

располагаясь внутри диапазона от 20 до 100 [3], а в случае $Z_{f}(p)$ вида (3) величина данного параметра ограничена интервалом $0,5 \ldots 2$ и находится как

$$
Q=\frac{1}{\omega_{0} T_{f}}=R_{f} \sqrt{\frac{C_{f}}{L_{f}}} .
$$

На рис. 2 изображены графические зависимости $\left|Z_{f}(j \omega)\right|$ рассмотренных выше пассивных фильтров высших гармоник, иллюстрирующих влияние $Q$ на участок АЧХ вблизи желаемой частоты $\omega_{0}$.

В качестве графической иллюстрации принципа действия пассивных ФКУ на рис. 3,4 изображены полученные в программном приложении «MATLAB-Simulink2 трехфазные токи и гистограмма частотного спектра питающей неуправляемый мостовой выпрямитель распределительной линии с параметрами

$$
R_{s}=3,6 \text { Ом, } L_{s}=5,8 \mathrm{м} \Gamma \mathrm{H},
$$

до и после включения двух резонансных контуров с $Z_{f}(p)$ вида (2), настроенных в соответствии с формулой (1) на 5-ю и 7-ю гармоники.

Как видно из результатов цифрового моделирования, пассивная фильтрация позволяет обеспечить приемлемый уровень высших гармоник в $i_{s}(t)$, амплитудные значения которых непосредственно зависят от температурного дрейфа параметров ФКУ, а также текущих вариаций частоты и конфигурации распределительной сети $[2,5]$. 

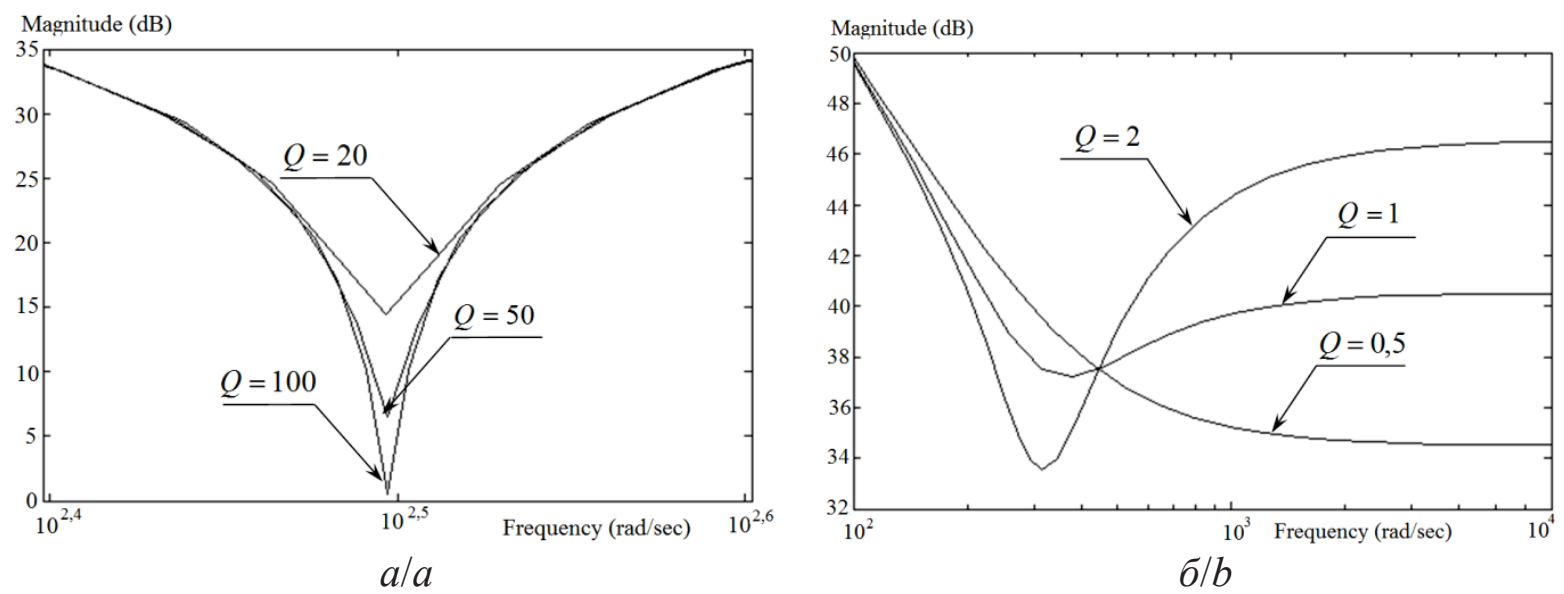

Pис. 2. ЛАЧХ полосового (а) и высокочастотного (б) фильтров второго порядка

Fig. 2. Asymptotic Bode magnitude plots of tuned ( $a$ ) and high-pass (b) second-order filters
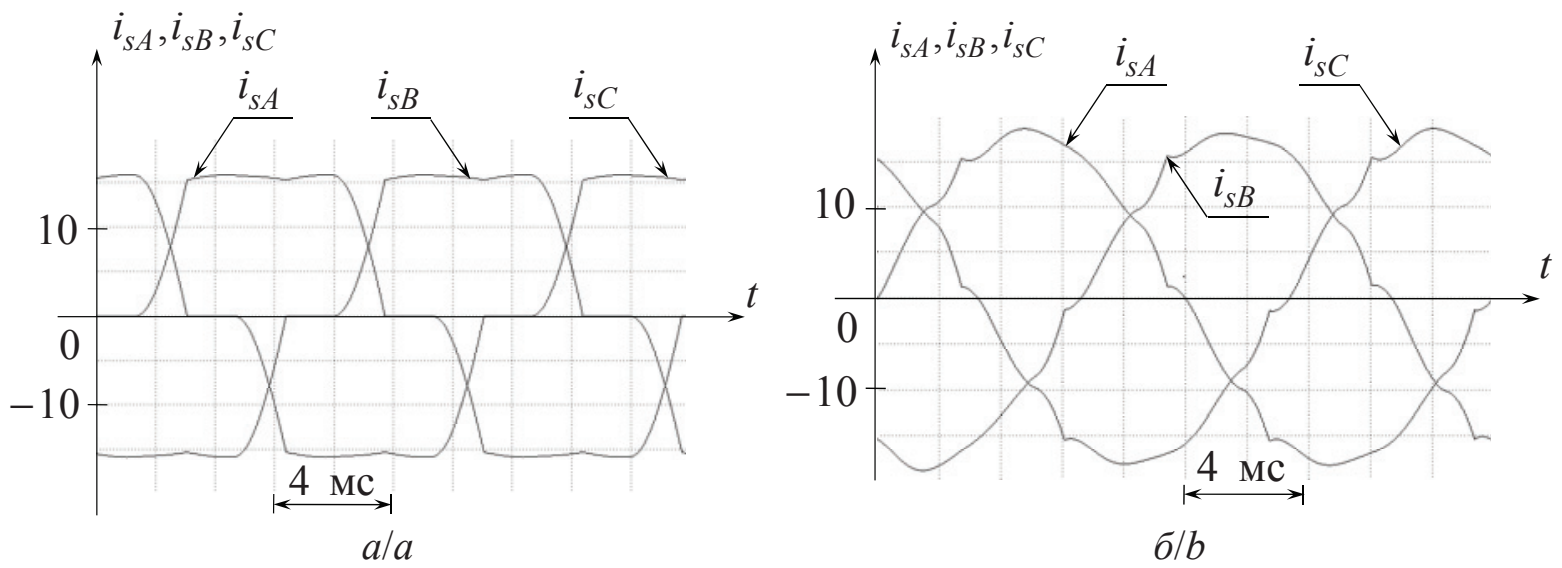

Рис. 3. Трехфазные сетевые токи до (а) и после (б) пассивной фильтраиии 5-й и 7-й гармоник

Fig. 3. Three-phase grid current waveforms before $(a)$ and after $(b)$ passive mitigation of $5^{\text {th }}$ and $7^{\text {th }}$ harmonics

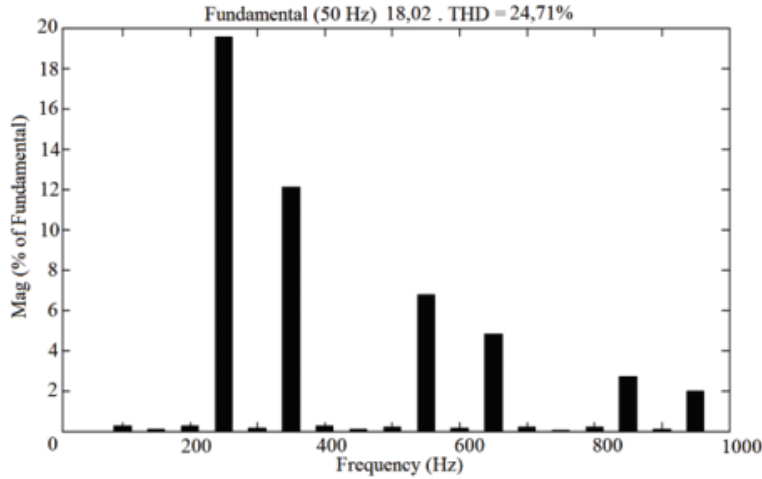

$a / a$

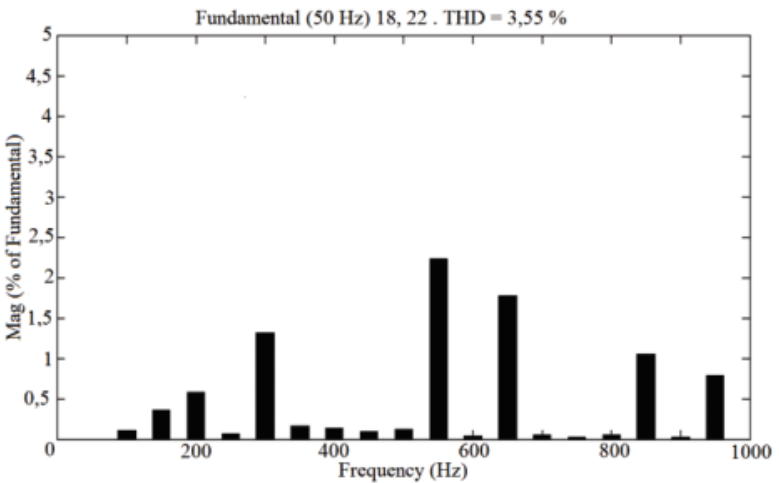

$\sigma / b$

Рис. 4. Спектральный состав сетевого тока в трехфазной системе с мостовым выпрямителем до (а) и после (б) пассивной фильтраиии 5-й и 7-й гармоник

Fig. 4. Harmonic content of three-phase grid currents for the bridge rectifier before (a) and after ( $b$ ) passive mitigation of $5^{\text {th }}$ and $7^{\text {th }}$ harmonics

\section{Активные фильтрокомпенсирующие устройства}

Активные силовые фильтры (АСФ), являющиеся в настоящее время наиболее эффективным техническим средством приведения реальных показателей качества электропитания к нормированным величинам, осуществляют непрерывную генерацию в систему электроснабжения сигналов компенсации сложной периодической формы, обратных по мгновенным значениям, как амплитудно-фазовым отклонениям, так и имеющим место искажениям. 
В зависимости от способа подключения данного типа ФКУ относительно трехфазной сети ТG и эксплуатируемого электрооборудования TL, силовые преобразовательные устройства активной фильтрации подразделяются [2, 3, 8, 9] на параллельные (shunt active power filter) и последовательные (series active power filter) АСФ, которые корректируют мгновенную форму сетевых токов и напряжений соответственно, а также их последовательнопараллельная комбинацию (unified power quality conditioner), что иллюстрирует рис. 5 , на котором используются следующие обозначения: CВ - система управления, осуществляющая на основании текущей информации с датчиков токов CS и напряжений VS формирование уставок $i_{f}^{*}$ и $u_{f}^{*}$ на компенсационные воздействия $i_{f}$ или $u_{f}$; VSI1, VSI2 - автономные инверторы с ШИМ-законом коммутации полупроводниковых ключей, первый из которых входит в состав последовательного АСФ, а второй параллельного ФКУ; C - емкостной накопитель электрической энергии; L - индуктивный дроссель, ограничивающий пульсации выходных токов $i_{f}$; Т - согласующий трансформатор однофазного или трехфазного исполнений.

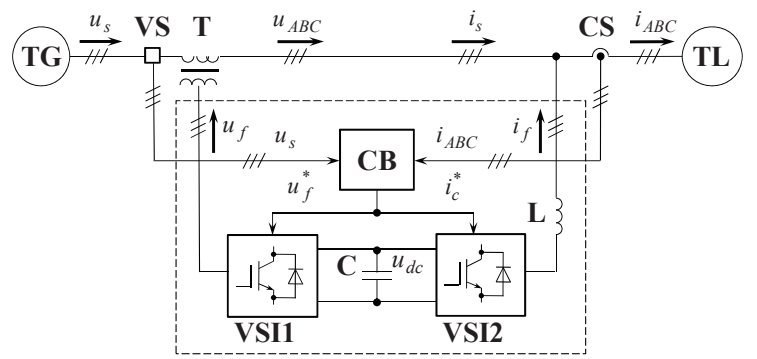

Рис. 5. Упрощенная функииональная схема последовательно-па раллельного АСФ

Fig. 5. Simplified bloc diagram of the series-shunt active power fil ters $(A P F)$

В отличие от рассмотренных выше пассивных RLC-цепей, исключающих из частотного спектра трехфазных токов только высшие гармоники, АСФ обладает более широким набором функциональных возможностей $[2,3,9]$, включающих в себя коррекцию углового сдвига, исключение активных потерь в нулевом проводе, демпфирование резонансных явлений и т. д.

Аналитическая процедура синтеза алгоритмов активной фильтрации основывается на предварительном выделении из общего потока электрической энергии в единицу времени неактивных составляющих мгновенной мощности в рамках векторного $[10,11]$ или алгебраического $[12,13]$ подходов к анализу энергетических характеристик трехфазных систем. Как отмечено в [14], в последнем случае удается значительно повысить быстродействие численного расчета $i_{f}^{*}$ или $u_{f}^{*}$ по отношению к структурам первого типа за счет сокращения общего количества выполняемых математических операций и отказа от линейных преобразова- ний исходного базиса, что в конечном итоге позволяет при прочих равных условиях снизить итоговые требования по производительности и объему памяти, которые предъявляются к программноаппаратной части системы управления.

В связи с тем, что параллельные АСФ обладают рядом преимуществ по отношению к последовательному типу активного ФКУ [3, 9], далее остановимся более подробно на законе компенсации в форме токов $i_{f}$ с использованием четырехмерного гиперкомплексного пространства, представив трехфазные переменные в следующем виде [15]

$$
\mathbf{X}_{A B C}=x_{A} \circ \mathbf{q}_{1}+x_{B} \circ \mathbf{q}_{2}+x_{C} \circ \mathbf{q}_{3} \text {, }
$$

здесь $x_{A}, x_{B}, x_{C}$ - мгновенные значения электрических величин; $\mathbf{q}_{1}, \mathbf{q}_{2}, \mathbf{q}_{3}$ - мнимые единицы, подчиняющиеся некоммутативным правилам произведения, в результате чего кватернион мгновенной мощности находится как [16]

$$
\mathbf{P}_{A B C}=\mathbf{U}_{A B C} \circ \mathbf{I}_{A B C}=\operatorname{Re} \mathbf{P}_{A B C}+\operatorname{Im} \mathbf{P}_{A B C},
$$

где $\operatorname{Re} \mathbf{P}_{A B C}, \operatorname{Im} \mathbf{P}_{A B C}$ - вещественная и мнимая части $\operatorname{Re} \mathbf{P}_{A B C}=p_{A B C} \circ 1 ; \operatorname{Im} \mathbf{P}_{A B C}=q_{A} \circ \mathbf{q}_{1}+q_{B} \circ \mathbf{q}_{2}+q \circ \mathbf{q}_{3}$, вещественные коэффициенты которых вычисляются в соответствии со следующими формулами

$$
\begin{gathered}
p_{A B C}=-u_{A} i_{A}-u_{B} i_{B}-u_{C} i_{C}, \\
q_{A}=u_{B} i_{C}-u_{C} i_{B}, q_{B}=u_{C} i_{A}-u_{A} i_{C}, q_{C}=u_{A} i_{B}-u_{B} i_{A} .
\end{gathered}
$$

Для математического описания трехфазных токов нагрузки $\mathbf{I}_{A B C}$ в функции $\mathbf{P}_{A B C}$ применяется обратный кватернион напряжений [14]

$$
\mathbf{U}_{A B C}^{-1}=-\left\|\mathbf{U}_{A B C}\right\|^{-1} \circ \mathbf{U}_{A B C},
$$

здесь $\left\|\mathbf{U}_{A B C}\right\|=\sum_{j=A, B, C} u_{j}^{2}-$ норма гиперкомплексного числа вида (4), с учетом которого становится справедливым [2]

$$
\mathbf{I}_{A B C}=\mathbf{U}_{A B C}^{-1} \circ\left(\operatorname{Re} \mathbf{P}_{A B C}+\operatorname{Im} \mathbf{P}_{A B C}\right)=\mathbf{I}_{A B C}^{p}+\mathbf{I}_{A B C}^{q},
$$

т. е. по аналогии с (5) $\mathbf{I}_{A B C}$ также раскладывается на активную (вещественную) $\mathbf{I}_{A B C}^{p}$ и реактивную (мнимую) $\mathbf{I}_{A B C}^{q}$ компоненты [15]

$$
\begin{gathered}
\mathbf{I}_{A B C}^{p}=\mathbf{U}_{A B C}^{-1} \circ \operatorname{Re} \mathbf{P}_{A B C}=i_{A}^{p} \circ \mathbf{q}_{1}+i_{B}^{p} \circ \mathbf{q}_{2}+i_{C}^{p} \circ \mathbf{q}_{3}, \\
\mathbf{I}_{A B C}^{q}=\mathbf{U}_{A B C}^{-1} \circ \operatorname{Im} \mathbf{P}_{A B C}= \\
=\left(i_{A}-i_{A}^{p}\right) \circ \mathbf{q}_{1}+\left(i_{B}-i_{B}^{p}\right) \circ \mathbf{q}_{2}+\left(i_{C}-i_{C}^{p}\right) \circ \mathbf{q}_{3},
\end{gathered}
$$

где $i_{A}^{p}, i_{B}^{p}, i_{C}^{p}-$ вещественные коэффициенты при мнимых единицах [13]

$$
i_{j}^{p}=-\left\|\mathbf{U}_{A B C}\right\|^{-1} \circ u_{j} \circ \operatorname{Re} \mathbf{P}_{A B C} .
$$

Как показывают представленные в [16] результаты аналитического исследования $\mathbf{P}_{A B C}$ в случае различного рода потребителей электрической энергии, параметрическая асимметрия, насыщение, нелинейные вольт-амперные характеристики элементов электрооборудования и т. д. приводят к образованию $\mathbf{I}_{A B C}^{q}$ и мультигармонических пульсаций в составе $\mathbf{I}_{A B C}^{p}$, вычисляемых как 


$$
\tilde{\mathbf{I}}_{A B C}^{p}=\mathbf{I}_{A B C}^{p}-\overline{\mathbf{I}}_{A B C}^{p},
$$

здесь $\overline{\mathbf{I}}_{A B C}^{p}=\mathbf{U}_{A B C}^{-1} \bar{p}_{A B C}-$ переменная составляющая $\mathbf{I}_{A B C}^{p}$, изменяющаяся во времени с частотой сети $\omega_{s}=2 \pi \cdot 50 \mathrm{c}^{-1}$, которая зависит от среднего значения $\mathrm{Re}_{\text {ABC }}$ и выступает в качестве эталона энергопотребления, в результате чего окончательно получаем следующий закон активной фильтрации [2]

$$
\begin{aligned}
\mathbf{I}_{f} & =\tilde{\mathbf{I}}_{A B C}^{p}+\mathbf{I}_{A B C}^{q}-\mathbf{U}_{A B C}^{-1} \circ \Delta \bar{p}= \\
& =\mathbf{I}_{A B C}-\overline{\mathbf{I}}_{A B C}^{p}-\mathbf{U}_{A B C}^{-1} \circ \Delta \bar{p},
\end{aligned}
$$

где $\Delta \bar{p}$ - стабилизирующая добавка, обеспечивающая поддержание на заданном уровне напряжения емкостного накопителя энергии $u_{d c}$, который, как отмечено в [2], при прочих равных условиях реализуется на практике с меньшим количеством выполняемых математических операций по отношению к традиционным структурам.

Для получения текущей информации о мгновенных значениях $\overline{\mathbf{I}}_{A B C}^{p}$ в (6) можно воспользоваться специальными динамическими подсистемами оценивания $[17,18]$ или определить данную гиперкомплексную переменную при помощи разложения трехфазных токов нагрузки в ряд Фурье [11]:

$$
i_{j}=\frac{B_{0}}{2}+\sum_{k=1}^{\infty}\left(A_{k} \sin \left(k \omega_{s} t\right)+B_{k} \cos \left(k \omega_{s} t\right)\right)
$$

где $B_{0}, A_{k}, B_{k}$ - коэффициенты, определяемые как

$$
\begin{gathered}
A_{k}=\frac{\omega_{s}}{\pi} \int_{0}^{2 \pi \omega_{s}^{-1}} i_{j} \sin \left(k \omega_{s} t\right) d t \text { при } k=1,2,3 \ldots, \\
B_{k}=\frac{\omega_{s}}{\pi} \int_{0}^{2 \pi \omega_{s}^{-1}} i_{j} \cos \left(k \omega_{s} t\right) d t \text { при } k=0,1,2,3 \ldots,
\end{gathered}
$$

и использования формул синуса и косинуса двойного угла, в соответствии с которыми становятся справедливы следующие соотношения

$$
\begin{gathered}
i_{j} \cos \omega_{s} t= \\
=\frac{B_{1}}{2}+\frac{B_{0}}{2} \cos \omega_{s} t+\frac{B_{1}}{2} \cos 2 \omega_{s} t+\frac{A_{1}}{2} \sin 2 \omega_{s} t+\ldots, \\
i_{j} \sin \omega_{s} t= \\
=\frac{A_{1}}{2}+\frac{B_{0}}{2} \sin \omega_{s} t+\frac{B_{1}}{2} \sin 2 \omega_{s} t-\frac{A_{1}}{2} \cos 2 \omega_{s} t+\ldots,
\end{gathered}
$$

здесь $\cos \omega_{s} t, \sin \omega_{s} t-$ тригонометрические функции углового сдвига напряжений сети $u_{A}, u_{B}, u_{C}$ частотой 50 Гц, получаемые на выходе специального контура фазовой автоподстройки в координатных осях $\alpha, \beta$ после преобразование Кларк (E. Clarke Transformation) [19].

Как видно из правой части последних равенств, амплитудные значения $A_{1}$ и $B_{1}$ основной гармоники в установившемся процессе представляют собой неизменные во времени величины, которые можно выделить из измеренного сигнала $i_{j}$ с помощью специальной подсистемы с фильтром низких частот ФНЧ, показанной на рис. 6.

В качестве графической иллюстрации фильтрующих свойств АСФ с алгоритмом управления вида (6), параметры которого представлены в таблице, на рис. 7,8 , изображены экспериментальные зависимости сетевых токов $i_{s A}, i_{s B}, i_{s c}$ и кватерниона мгновенной мощности $\mathbf{P}_{A B C}$ трехпроводной $\mathrm{RL}$-нагрузки с отсекающим диодом в фазе $B$, вещественные коэффициенты мнимой части которого равны друг другу [13]

$$
q_{A}=q_{B}=q_{C} .
$$

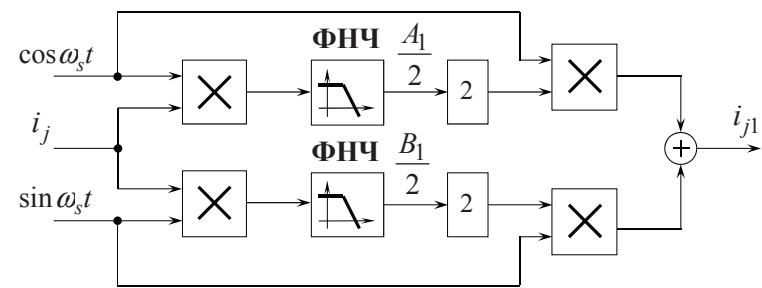

Pис.6. Функииональная схема получения основной гармоники фазного тока нагрузки $i_{j 1}$

Fig. 6. Block diagram for obtaining fundamental harmonic of load phase current $i_{\mathrm{j} 1}$

таблица. Параметры АСФ

Table. APF parameters

\begin{tabular}{|l|c|}
\hline \multicolumn{1}{|c|}{$\begin{array}{c}\text { Параметр силовой части АСФ } \\
\text { Роwer unit parameter }\end{array}$} & $\begin{array}{c}\text { Значение } \\
\text { Value }\end{array}$ \\
\hline $\begin{array}{l}\text { Емкость накопителя электрической энергии } \\
\text { Electric energy storage сарасity }\end{array}$ & 1,65 мФ (mF) \\
\hline $\begin{array}{l}\text { Индуктивность выходных дросселей } \\
\text { Inductance of the output inductors }\end{array}$ & $9,4 \mathrm{м \Gamma н} \mathrm{(mH)}$ \\
\hline $\begin{array}{l}\text { Частота коммутации IGBT-модулей инвертора } \\
\text { Switching frequency of IGBT modules of the inverter }\end{array}$ & 2,7 кГц (kHz) \\
\hline $\begin{array}{l}\text { Коэффициент датчика напряжения } \\
\text { Voltage sensor ratio }\end{array}$ & 0,01 o.e. $($ p.u. $)$ \\
\hline Коэффициент датчика тока/Current sensor ratio & $0,18 \mathrm{BA}^{-1}\left(\mathrm{VA}^{-1}\right)$ \\
\hline
\end{tabular}

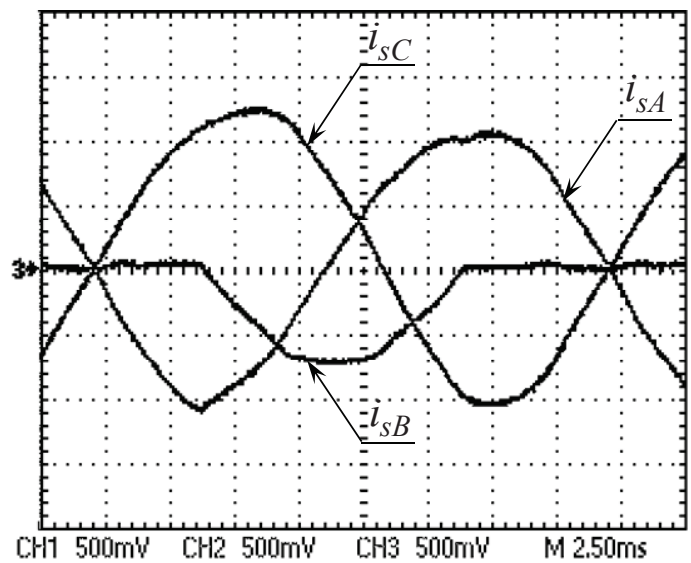

Puc. 7. Трехфазные токи сети при питании нелинейной $R L$-нагрузки

Fig. 7. Grid three-phase currents in case of the nonlinear RL-load

В свою очередь на рис. 9 приведены осциллограммы аналогичных переменных $i_{s A}, i_{s B}, i_{s c}$ до и после вступления в работу параллельного АСФ, сопровождаемого одновременным зарядом силовой емкости С от начального значения 340 В до величины $475 \mathrm{~B}$, которые иллюстрируют динамиче- 

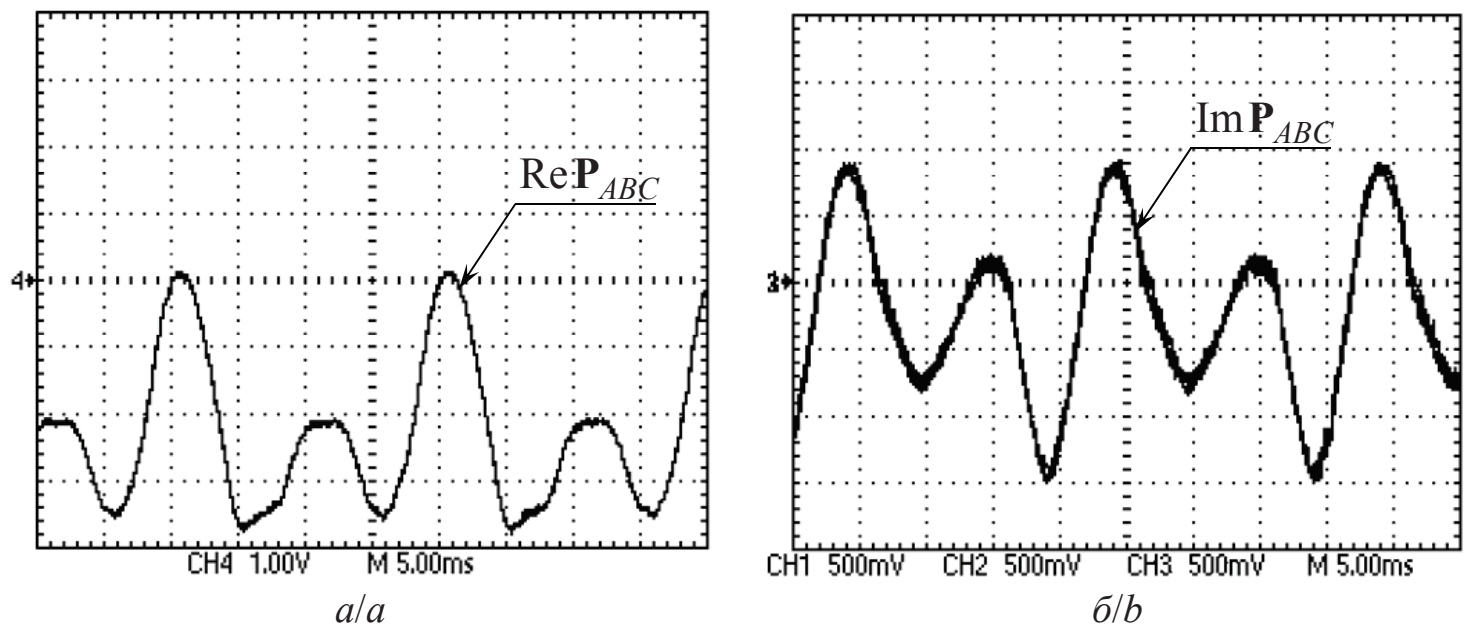

Pис. 8. Вещественная (а) и мнимая (б) части кватерниона мгновенной мощности

Fig. 8. Real ( a) and complex (b) components of the instantaneous power quaternion

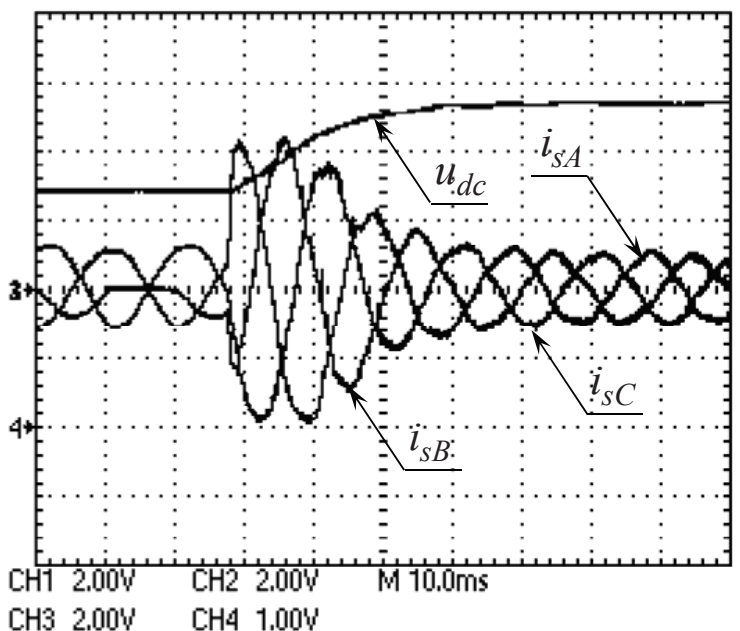

$a / a$

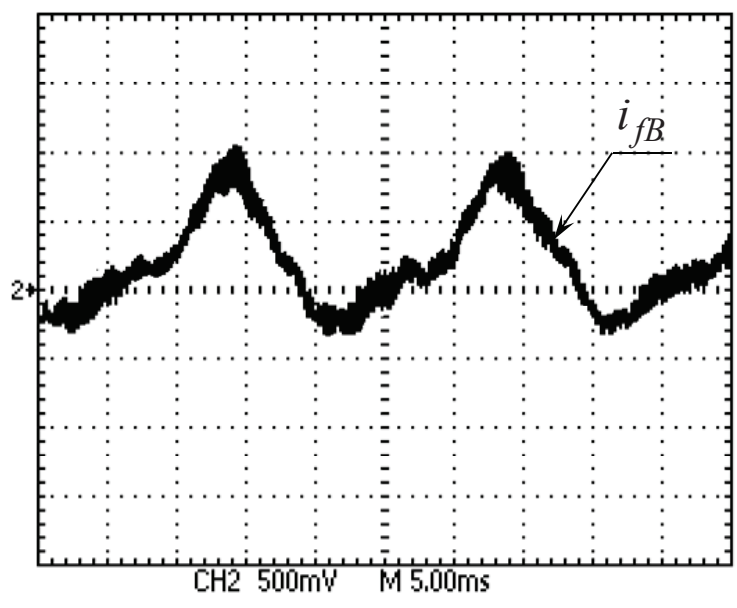

$\sigma / b$

Pис.9. Сетевые токи $i_{s A}, i_{s B}, i_{s c}$, напряжение $u_{d c}$ и ток компенсаиии $i_{f B}$ в трехфазной системе с $А С \Phi$

Fig. 9. Grid currents $i_{\mathrm{s} A}, i_{\mathrm{sB}}, i_{s c}, D C$ link voltage $u_{d c}$ and compensating current $i_{f B}$ in the three-phase system with APF

ские характеристики АСФ, а также соответствующее компенсационное воздействие фазы $B$.

При этом также необходимо отметить, что для снижения влияния высокочастотных пульсаций $i_{f}$ на гармонический состав сигналов распределительной сети вследствие дискретного характера работы автономного инвертора АСФ, на его выходе дополнительно устанавливается пассивный фильтр LCL- или LLCL-топологий [20].

\section{Заключение}

На основании представленных в данной статье кратких сведений о силовых устройствах пассивной и активной фильтрации высших гармоник можно заключить, что практическое применение ФКУ позволяет увеличить пропускную способность линий электропередачи и до 30 \% уменьшить среднеквадратичные потери при передаче, распределении и преобразовании электрической энергии переменного тока за счет частичной или полной компенсации неэффективных составляющих мгновенной мощности, вызванных асимметрией и нелинейностью процессов в электрооборудовании технологических машин [21]. При этом включение в систему электроснабжения АСФ является более предпочтительным по отношению к шунтирующим RLC-цепям соответствующего порядка, т. к. в этом случае достигается полная инвариантность $\mathrm{\kappa}$ температурному дрейфу параметров, осуществляется автоматическая подстройка алгоритма управления при изменении режимов работы нагрузки или конфигурации распределительной сети, а также полностью отсутствуют какие-либо ограничения по качественному составу электрической энергии.

Исследование выполнено в рамках програмлы «Разработка эффективного устройства нового поколения для компенсации реактивной мощности и подавления гармонических составляющих токов высших порлдков в электроэнергетике», гос. задание № 8.10997.2018/11.12. 


\section{СПИСОК ЛИТЕРАТУРЫ}

1. Методика расчета электромагнитных процессов в многоуровневых полупроводниковых преобразователях для электротехнического оборудования горнодобывающей промышленности / Б.Ф. Симонов, М.А. Дыбко, С.В. Брованов, С.А. Харитонов // Физико-технические проблемы разработки полезных ископаемых. СО РАН. - 2015. - № 2. - С. 97-110.

2. Нос 0.В. Методы анализа и синтеза трехфазных систем с активными силовыми фильтрами в гиперкомплексном пространстве: дис.... д-ра техн. наук. - Новосибирск, 2015. - 385 с.

3. Akagi H. Active harmonic filters // Proceedings of the IEEE. 2005. - V. 93. - № 12. - P. 2128-2141.

4. Phipps J.K. A transfer function approach to harmonic filter design // IEEE Industry Applications Magazine. - 1997. - V. 3. P. 68-82.

5. Das J.C. Passive filters - potentialities and limitations // IEEE Transactions on Industry Applications. - 2004. - V. 40. - № 1. P. 232-241

6. Nassif A.B., Xu W. Passive harmonic filters for medium-voltage industrial systems: practical considerations and topology analysis // The $39^{\text {th }}$ North American Power Symposium Proceedings. Las Cruces, New Mexico, USA, September 30 - October 2, 2007. P. 301-307.

7. Gonzalez D.A., McCall J.C. Design of filters to reduce harmonic distortion in industrial power systems // IEEE Transactions on Industry Applications. - 1987. - V. IA-23. - № 3. - P. 504-512.

8. Khadkikar V. Enhancing electric power quality using UPQC: a comprehensive overview // IEEE Transactions on Power Electronics. - 2012. - V. 27. - № 5. - P. 2284-2297.

9. Akagi H., Watanabe E.H., Aredes M. Instantaneous power theory and applications to power conditioning. 2 ed. - Hoboken, New Jersey, USA: John Wiley \& Sons, Inc., 2017. - 450 p.

10. Willems J.L. Mathematical foundations of the instantaneous power concepts: a geometrical approach // European Transactions on Electrical Power. - 1996. - V. 6. - № 5. - P. 299-304.

11. Herrera R.S., Salmeron P., Kim H. Instantaneous reactive power theory applied to active power filter compensation different approaches, assessment, and experimental results // IEEE Transactions on Industrial Electronics. - 2008. - V. 55. - № 1. P. 184-196.

12. Cristaldi L., Ferrero A. Mathematical foundations of the instantaneous power concepts: an algebraic approach // European Transactions on Electrical Power. - 1996. - V. 6. - № 5. - P. 305-309.
13. Nos 0.V. Control strategy of shunt active power filter based on an algebraic approach // The 16 International conference of young specialists on micro/nanotechnologies and electron devices, EDM 2015: proc. - Altai, Erlagol, 29 June - 3 July 2015. - Novosibirsk: NSTU, 2015. - P. 459-463.

14. Nos O.V., Brovanov S.V., Dybko M.A. Development of active filtering algorithms for higher harmonics in electrical power circuits // Optoelectronics, Instrumentation and Data Processing. 2016. - V. 52. - № 6. - P. 557-562.

15. Brasil V.P., Filho A. de L.F., Ishihara J.Y., Electrical three phase circuit analysis using quaternions // The $18^{\text {th }}$ International Conference on Harmonics and Quality of Power (ICHQP). - Ljubljana, Slovenia, 13-16 May, 2018. - P. 1-6.

16. Nos 0.V., Dudin A., Petzoldt J. The instantaneous power quaternion of the three-phase electric circuit with linear load // The 17 International conference of young specialists on micro/nanotechnologies and electron devices, EDM 2016: proc. - Altai, Erlagol, 30 June - 4 July 2016. - Novosibirsk: NSTU, 2016. P. 526-531.

17. A method to improve the dynamic performance of moving average filter based PLL / J. Wang, J. Liang, F. Gao, L. Zhang, Z. Wang // IEEE Transactions on Power Electronics. - 2015. V. 30. - № 10. - P. 5978-5990.

18. Fault-decoupled instantaneous frequency and phase angle estimation for three-phase grid-connected inverters / G. de Donato, G. Scelba, G. Borocci, F.G. Capponi, G. Scarcella // IEEE Transactions on Power Electronics. - 2016. - V. 31. - № 4. P. 2880-2889.

19. Duesterhoeft W.C., Schulz M.W., Clarke E. Determination of instantaneous currents and voltages by means of alpha, beta, and zero components // Transactions of the American Institute of Electrical Engineers. - 1951. - V. 70. - № 2. - P. 1248-1255.

20. LLCL-filtered grid converter with improved stability and robustness / M. Huang, X. Wang, P.C. Loh, F. Blaabjerg // IEEE Transactions on Power Electronics. - 2016. - V. 31. - № 5. P. 3958-3967.

21. Паймухин Е.Т. Энергоэффективность применения динамического фильтро-компенсирующего устройства ДФКУ в 000 «РН-ПУРНЕФТЕГАЗ» // Инженерная практика. - 2011. № 6. - С. 58-60.

Поступила 26.02.2019 2.

\section{Информация об авторах}

Нос O.B., доктор технических наук, профессор кафедры проектирования технологических машин Новосибирского государственного технического университета.

Востриков A.C., доктор технических наук, профессор кафедры автоматики Новосибирского государственного технического университета.

Штанг A.A., кандидат технических наук, доцент кафедры электротехнических комплексов Новосибирского государственного технического университета,.

Малявко E.Ю., аспирант Новосибирского государственного технического университета. 
UDC 621.314.2-729.3

\section{ENHANCING ENERGY EFFICIENCY OF INDUSTRIAL PRODUCTION BY USING POWER HARMONIC FILTERS}

Oleg V. Nos',

nos@corp.nstu.ru

Anatoliy S. Vostrikov',

vostrikov@sintez.nstu.ru

Alexandr A. Shtang',

shtang@corp.nstu.ru

Ekaterina Yu. Malyavko',

arteb@smc.nstu.ru

1 Novosibirsk State Technical University,

20, Karl Marx avenue, Novosibirsk, 630073, Russia.

Relevance. At present time many technological processes in oil and gas industry sector apply the high-performance three-phase equipments with power electronic converters such as variable-frequency AC drives or uninterruptible power supplies which are nonlinear loads and can cause various problems in distribution systems, including voltage and current unbalance, resonances phenomena, resistance current losses in the neutral wire, overheating of motors and transformers etc. In order to improve the electromagnetic compatibility of different power consumers in the industrial power supply systems the passive capacitor compensators for reactive power correction are usually used, that are ineffective in case of nonlinear loads. Furthermore this equipment generates the reactive power even if any loads are absent and do not meet modern requirements in area of power conditioning. To avoid the impact of the above-mentioned drawbacks on normal operation conditions of three-phase systems the development of novel technical approaches to power circuit design, methods for analyzing of electrical power processes and control strategies for power filtering are needed that provide the desired power quality in the low voltage and medium voltage distribution grids regardless of the load electrical circuits.

The aim of the research is a comprehensive analysis of existing approaches to electromagnetic compatibility improvement of the threephase loads in the distribution AC system and description of the main restrictions of power compensators under normal operation conditions for industrial applications in mineral resources sector; the control design for enhancing an operational stability of active power filters under time-varying parameter disturbances as well as variations of load behaviors or distribution system configuration.

Objects: AC electrical power supply systems with power electronics converters and load units in which there are distortions in the instantaneous sinusoidal waveforms of grid three-phase variables; passive and active power filters for high-frequency harmonic correction. Methods: non-commutative algebra of quaternion; four-dimensional hyper-complex space representation; methods of spectral analysis and Fourier series decomposition.

Results: the brief survey of different approaches and techniques to the power quality enhancing in the AC electrical power supply systems of mineral resources industrial sector and control strategy of active power filters that will provide balanced or/and sinusoidal grid currents in the three-phase systems with the zero or leading/lagging phase shift relative to the corresponding voltage.

\section{Key words:}

Nonlinear electromagnetic processes, energy efficiency, power compensators, series-shunt active power filters, hyper-complex numbers.

The research is performed within the program «Development of an energy effective device for compensation of reactive power and suppression of high-order harmonic components in the power systems», public research program of Russian Federation No. 8.10997.2018/11.12.

\section{REFERENCES}

1. Simonov B.F., Dybko M.A., Brovanov S.V., Kharitonov S.A. Calculation procedure for electromagnetic processes in multilevel semiconductor converters for electrical equipment in mining. Jour nal of Mining Science, 2015, vol. 51, no. 2, pp. 280-291.

2. Nos 0.V. Metody analiza i sinteza trekhfaznykh system saktivnymi silovymi filtrami v giperkompleksnom prostranstve. Dis. Dokt. nauk [Analysis and design methods of three-phase systems with active power filters in the hyper-complex space. Dr. Diss.]. Novosibirsk, 2015. $385 \mathrm{p}$.

3. Akagi H. Active harmonic filters. Proceedings of the IEEE, 2005, vol. 93 , no. 12, pp. 2128-2141.

4. Phipps J.K. A transfer function approach to harmonic filter design. IEEE Industry Applications Magazine, 1997, vol. 3, pp. $68-82$.
5. Das J.C. Passive filters - potentialities and limitations. IEEE Transactions on Industry Applications, 2004, vol. 40, no. 1, pp. 232-241.

6. Nassif A.B., Xu W. Passive Harmonic filters for medium-voltage industrial systems: practical considerations and topology analysis. The $39^{\text {th }}$ North American Power Symposium Proceedings. Las Cruces, New Mexico, USA, September 30 - October 2, 2007. pp. 301-307.

7. Gonzalez D.A., McCall J.C. Design of filters to reduce harmonic distortion in industrial power systems. IEEE Transactions on Industry Applications, 1987, vol. IA-23, no. 3, pp. 504-512.

8. Khadkikar V. Enhancing electric power quality using UPQC: a comprehensive overview. IEEE Transactions on Power Electronics, 2012, vol. 27, no. 5, pp. 2284-2297.

9. Akagi H., Watanabe E.H., Aredes M. Instantaneous power theory and applications to power conditioning. $2^{\text {nd }}$ ed. Hoboken, New Jersey, USA, John Wiley \& Sons, Inc., 2017. 450 p. 
10. Willems, J.L. Mathematical foundations of the instantaneous power concepts: a geometrical approach. European Transactions on Electrical Power, 1996, vol. 6, no. 5, pp. 299-304.

11. Herrera R.S., Salmeron P., Kim H. Instantaneous reactive power theory applied to active power filter compensation different approaches, assessment, and experimental results. IEEE Transactions on Industrial Electronics, 2008, vol. 55, no. 1, pp. 184-196.

12. Cristaldi L., Ferrero A. Mathematical foundations of the instantaneous power concepts: an algebraic approach. European Transactions on Electrical Power, 1996, vol. 6, no. 5, pp. 305-309.

13. Nos 0.V. Control strategy of shunt active power filter based on an algebraic approach. The 16 International conference of young specialists on micro/nanotechnologies and electron devices, EDM 2015: proc. Altai, Erlagol, 29 June - 3 July 2015. Novosibirsk, NSTU, 2015. pp. 459-463.

14. Nos 0.V., Brovanov S.V., Dybko M.A. Development of active filtering algorithms for higher harmonics in electrical power circuits. Optoelectronics, Instrumentation and Data Processing, 2016, vol. 52, no. 6, pp. 557-562.

15. Brasil V.P., Filho A. de L.F., Ishihara J.Y., Electrical three phase circuit analysis using quaternions. The $18^{\text {th }}$ International Conference on Harmonics and Quality of Power (ICHQP). Ljubljana, Slovenia, 13-16 May, 2018. pp. 1-6.

16. Nos 0.V., Dudin A., Petzoldt J. The instantaneous power quaternion of the three-phase electric circuit with linear load. The 17 In- ternational conference of young specialists on micro/nanotechnologies and electron devices, EDM 2016: proc. Altai, Erlagol, 30 June - 4 July 2016. Novosibirsk, NSTU, 2016. pp. 526-531.

17. Wang J., Liang J., Gao F., Zhang L., Wang Z. A method to improve the dynamic performance of moving average filter based PLL. IEEE Transactions on Power Electronics, 2015, vol. 30, no. 10, pp. 5978-5990.

18. Donato de G., Scelba G., Borocci G., Capponi F.G., Scarcella G. Fault-decoupled instantaneous frequency and phase angle estimation for three-phase grid-connected inverters. IEEE Transactions on Power Electronics, 2016, vol. 31, no. 4, pp. 2880-2889.

19. Duesterhoeft W.C., Schulz M.W., Clarke E. Determination of instantaneous currents and voltages by means of alpha, beta, and zero components. Transactions of the American Institute of Electrical Engineers, 1951, vol. 70, no. 2, pp. 1248-1255.

20. Huang M., Wang X., Loh P.C., Blaabjerg F. LLCL-filtered grid converter with improved stability and robustness. IEEE Transactions on Power Electronics, 2016, vol. 31, no. 5, pp. 3958-3967.

21. Paymukhin E.T. Energy efficiency of application of dynamic filtering and compensating device DFCD at RN-Purneftegaz. Engineering practice, 2011, no. 6, pp. 58-60. In Rus.

Received: 26 February 2019.

Information about the authors

Oleg V. Nos, Dr. Sc., professor, Novosibirsk State Technical University.

Anatoliy S. Vostrikov, Dr. Sc., professor, Novosibirsk State Technical University.

Alexandr A. Shtang, Cand. Sc., associate professor, Novosibirsk State Technical University.

Ekaterina Yu. Malyavko, postgraduate student, Novosibirsk State Technical University. 